\title{
Sarcoma de Kaposi asociado al VIH/SIDA: importancia de su diagnóstico para un correcto manejo odontológico.
}

\author{
HIV/AIDS associated Kaposi's sarcoma: importance of its \\ diagnosis for a correct odontologic management.
}

\author{
Gustavo Pedro Díaz Colmenares,* Anabell Alvarado Sánchez, ${ }^{\ddagger}$ Ana Isabel de la Garza, ${ }^{\S}$ Daniel A Guerra Leal"
}

\section{RESUMEN}

El sarcoma de Kaposi (SK) es una neoplasia vascular maligna poco frecuente, asociada al virus herpes humano tipo 8. Existen cuatro formas clínicas: clásico, endémico, asociado con inmunosupresión iatrogénica y asociado al VIH/SIDA. Este artículo presenta una revisión de la literatura sobre la epidemiología, la patogénesis, las manifestaciones clínicas y el tratamiento del sarcoma de Kaposi asociado al VIH/ SIDA (SK-VIH/SIDA) a propósito de un caso clínico manejado en la Clínica Académica de Atención Dental. La baja en la incidencia de esta neoplasia da lugar al desconocimiento de sus manifestaciones clínicas. En adición, los pacientes seropositivos suelen no mencionar su padecimiento en la anamnesis, lo cual representa un riesgo tanto para el paciente en su diagnóstico y manejo odontológico como para el odontólogo y el personal clínico con riesgo de contagio.

Palabras clave: Sarcoma, Kaposi, VIH/SIDA, neoplasia, cavidad oral, bioseguridad.

\section{ABSTRACT}

Kaposi sarcoma (KS) is an uncommon malignant vascular neoplasm, associated with human herpes virus type 8. There are four clinical presentations: classic, endemic, associated with iatrogenic immunosuppression and associated with AIDS. This article presents a review of the literature on epidemiology, pathogenesis, clinical manifestations, treatment and HIV/AIDS-associated Kaposi sarcoma (SK-HIV/AIDS) regarding a clinical case managed at the Academic Center of Dental Care. The decrease in the incidence of this neoplasm, leads to ignorance of its clinical manifestations. In addition, seropositive patients usually don't mention their condition in the anamnesis, which represents a risk for the patients on their diagnosis and the case management as well as for the dentist and the clinical personnel from risk of infection.

Keywords: Sarcoma, Kaposi, HIV/AIDS, neoplasia, oral cavity, biosafety.

\section{INTRODUCCIÓN}

$E^{\prime}$ sarcoma de Kaposi (SK) es una neoplasia maligna angioproliferativa, multicéntrica, de origen endotelial asociada al virus del herpes humano 8 ( $\mathrm{VHH}-8)$. La infección por el $\mathrm{VHH}-8$ es un factor necesario para el desarrollo del SK; sin embargo, no es suficiente por sí solo, necesita de un cofactor como la infección por el virus de inmunodeficiencia humana $(\mathrm{VIH}) .^{1}$ La aparición del SK está relacionada con los conteos de linfocitos T CD4 en la sangre periférica, los cuales se manifiestan con una cuenta menor a 200 células por $\mathrm{mm}^{3} .^{2}$ Cabe recalcar que una

\footnotetext{
* Médico Cirujano Odontólogo pasante.

‡ Alumna de noveno semestre de la Carrera Médico Cirujano Odontólogo.

$\S$ Médico Cirujano Odontólogo.

\| Cirujano Maxilofacial, Coordinador del Centro Académico de Atención Odontológica (CAAD). Escuela de Medicina y Ciencias de la Salud.
}

Tecnológico de Monterrey.

Recibido: 10 Diciembre 2019. Aceptado para publicación: 29 Marzo 2020. 
cantidad igual o menor a 200 en la cuenta de linfocitos es característica para poder denominar que una persona se encuentra en la fase terminal de la infección del VIH, presentando manifestaciones clínicas, lo que es denominado como síndrome de inmunodeficiencia adquirida (SIDA). ${ }^{3}$

Existen cuatro variantes clínicas del sarcoma de Kaposi: la forma clásica, la endémica (África subsahariana), la asociada con pacientes inmunosuprimidos y la epidémica o asociada al VIH/SIDA. ${ }^{4}$ La primera de estas variantes fue descrita en 1872 por Moritz Kaposi y se observa comúnmente en varones de origen mediterráneo o judío alrededor de la sexta década de la vida. ${ }^{5}$ El SK endémico afecta a personas de regiones africanas y constituye el $9 \%$ de todas las tumoraciones malignas del área. ${ }^{6}$ La inmunosupresión a largo plazo en pacientes receptores de trasplantes de órganos aumenta 100 veces la probabilidad de desarrollar SK comparado con la población general, presentándose de 0.06 a $4.1 \%$ de los pacientes con trasplantes renales. ${ }^{7}$ Por último, el SK epidémico o asociado al VIH/SIDA es el más común, debido al advenimiento de la epidemia del síndrome de inmunodeficiencia adquirida (SIDA); sin embargo, su incidencia se ha reducido gracias a las terapias antirretrovirales. ${ }^{8}$

En más de 20\% de los casos de SK la cavidad oral es el sitio inicial de la manifestación clínica. ${ }^{9}$ También representa uno de los primeros signos de la progresión del virus del VIH al SIDA, lo cual eleva la tasa de mortalidad de dichos pacientes. ${ }^{10}$

\section{Manifestaciones clínicas}

Las manifestaciones clínicas del SK asociado al SIDA en la cavidad oral afectan, por lo regular, sitios de mucosa queratinizada, los más comunes son el paladar duro, la encía insertada y el dorso de la lengua. ${ }^{11}$ No obstante, puede presentarse en cualquier lugar de la cavidad oral, incluyendo el músculo masetero, la úvula y la orofaringe. ${ }^{12}$

Las lesiones varían de acuerdo a su tamaño y grado de desarrollo. En pacientes con SIDA, el tumor comienza como máculas eritematosas de color rojizo a violáceo poco circunscritas. ${ }^{13}$ Éste va progresando conforme el conteo de linfocitos CD4 va disminuyendo. ${ }^{14}$ Por lo que puede convertirse en lesiones con forma de placa o nodulares de color rojizo-café debido a depósitos de hemosiderina. ${ }^{13}$ Cuando la lesión es en forma de placa usualmente es asintomática. Por otro lado, la forma exofítica está asociada con supuración, sangrado y dolor si ésta se ulcera o infecta debido al trauma masticatorio. ${ }^{12}$ Las lesiones tienden a oscurecer en color y a fusionarse con lesiones adyacentes, formando clusters o racimos. ${ }^{9}$
Su tamaño varía de 5 a $22 \mathrm{~mm} \cdot{ }^{15}$ Cuando la encía está involucrada, el desarrollo de la lesión produce resorción del hueso alveolar y, por consiguiente, movilidad de los órganos dentarios implicados. ${ }^{16}$

El diagnóstico diferencial depende de la morfología de la lesión respecto al estadio de desarrollo que se encuentre. En etapas tempranas puede confundirse fácilmente con un hematoma, equimosis, pigmentación de melanina, pigmentación por amalgama o gingivitis localizada. ${ }^{13} \mathrm{En}$ su forma más avanzada, el diagnóstico diferencial debe incluir: granuloma piógeno, nevo oral, granuloma central de células gigantes, angiomatosis bacilar, absceso periodontal, hemangioma, hemangiosarcoma, linfangioma y otras neoplasias malignas asociadas al SIDA como el linfoma no-Hodgkin. Para realizar el diagnóstico definitivo es necesario el estudio histopatológico. ${ }^{17}$

\section{Tratamiento del SK-VIH}

El objetivo del tratamiento es mitigar los síntomas y mejorar el estado de salud general del paciente. Además del tratamiento sistémico, suele ser necesaria la atención bucodental dado a la amplia sintomatología oral que se presenta en el SK, tales como sangrado gingival, movilidad dental, pérdida dental, interferencia fonética y de la masticación, así como retención dentaria. ${ }^{18}$ En ocasiones se busca la mejoría estética en caso de ser necesario. ${ }^{19}$

El VHH-8 se expresa en una fase latente en el SK-VIH, por lo que antivirales como el cidofovir, foscarnet, ganciclovir o valganciclovir no tienen efecto alguno sobre la patología. ${ }^{20,21}$ Por otro lado, algunos fármacos causan una disminución pero no una erradicación total del SK,, $2-24$ por lo que no son curativos y su uso varía dependiendo de la extensión de la lesión, sus manifestaciones orales y el tipo epidemiológico.

La terapia local es la primera elección cuando el sarcoma no está relacionado con el VIH o el SIDA, dado a sus escasos efectos secundarios. Por el contrario, al estar limitado a la piel de manera superficial, la escisión quirúrgica, electroquimioterapia y la administración de agentes esclerosantes son las más convenientes en primera instancia, ${ }^{25}$ aunque el uso concomitante de tratamientos sistémicos como la terapia antirretroviral de gran actividad (TARGA o HAART, por sus siglas en inglés) ha demostrado resultados exitosos. El Instituto Nacional de Cáncer de los Estados Unidos ( $\mathrm{NCl}$ ) describe a la TARGA como el uso de tres o más agentes antirretrovirales, ${ }^{26}$ los cuales actúan en diferentes etapas del ciclo vital del VIH (Figura 1).

Se han descrito diversos tipos de abordajes para el tratamiento, ya sea de manera local, sistémica o mixta 


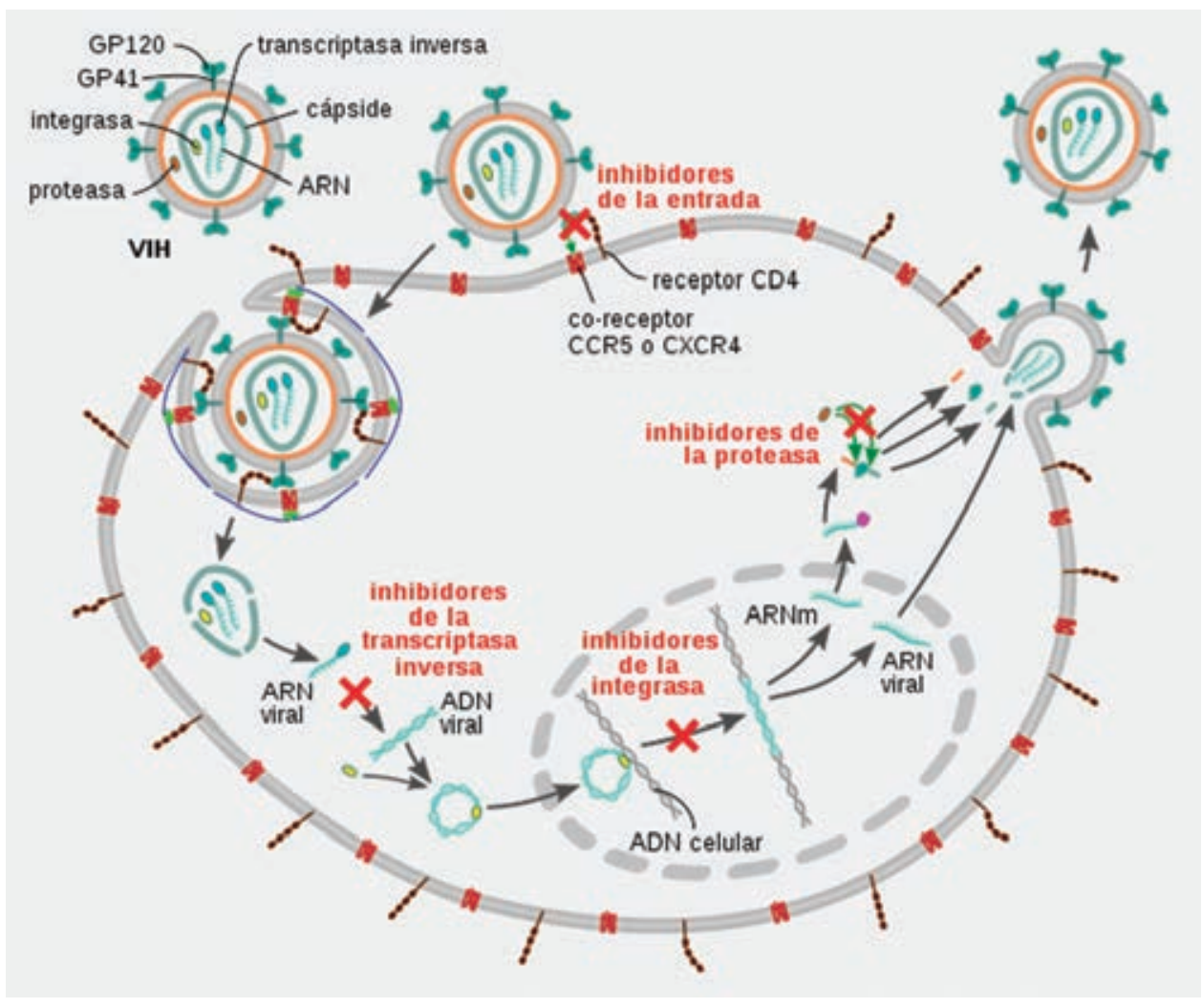

Figura 1:

Descripción de las etapas en las que actúan los antirretrovirales. ${ }^{27}$

(Figura 2). A pesar de la radiosensibilidad de las células del SK-VIH, no se recomienda la radioterapia en lesiones de la cavidad oral, dado a complicaciones como mucositis, xerostomía e incluso atentar contra la vida del paciente. ${ }^{19,28}$ Asimismo, se recomienda que la terapia sistémica con quimioterapia se inicie en una etapa maculopapular aunado a un conteo de CD4+ alto para prevenir que avance a una etapa exofítica. ${ }^{29}$

Cabe destacar la mención del síndrome de reconstitución inmunológica (SRI), el cual es una consecuencia adversa del tratamiento con TARGA; se caracteriza por la potencialización de infecciones oportunistas o subclínicas ya existentes en el paciente e incluso puede causar problemas mortales. ${ }^{30-32}$ Este fenómeno ha tenido mayor relevancia en pacientes con $\mathrm{VIH}$ a diferencia de pacientes inmunosuprimidos por trasplantes, quimioterapia o infecciones. ${ }^{31,33,34}$

\section{PRESENTACIÓN DEL CASO}

\section{Historia clínica del paciente}

Paciente masculino de 21 años de edad se presenta al Centro Académico de Atención Dental (CAAD) del Tec- nológico de Monterrey en la ciudad de Monterrey, debido a un aumento de volumen doloroso en región vestibular del cuadrante superior derecho. El paciente refiere haber sido sometido en dos ocasiones a drenajes de la lesión en diferentes clínicas, pensando que se trataba de un absceso periodontal o endodontal.

En el interrogatorio por aparatos y sistemas el paciente refiere ser $\mathrm{VIH}$ seropositivo con tres años de evolución y, al momento de la consulta, el sujeto desconocía tener manifestaciones del SIDA. También, menciona estar siendo tratado con Atripla (efavirenz $600 \mathrm{mg}+$ emtricitabine $200 \mathrm{mg}+$ tenofovir disoproxil fumarate $300 \mathrm{mg}$ ) y sulfametoxazol $800 \mathrm{mg}+$ trimetoprim $160 \mathrm{mg}$. Además, indica ser alérgico a las penicilinas y ciprofloxacino.

\section{Hallazgos clínicos y radiográficos}

En la exploración física extraoral se observa aumento de volumen en región nasogeniana de lado derecho y lesiones papulosas violáceas de $1 \times 1 \mathrm{~cm}$ de tamaño que se extienden en cuello, cara y punta de la nariz (Figura $3 A)$. Dentro de la exploración intraoral se encuentra un 
aumento de volumen en región vestibular, a nivel de órganos dentarios 1.4 y 1.5, bilobular, de consistencia dura y con dolor a la palpación (Figura 3B). Al sondeo periodontal se observan profundidades entre $4-5 \mathrm{~mm}$ sin recesiones gingivales, sangrado y movilidad dental grado 2. Además, se observa lesión de coloración violácea en paladar duro del lado derecho con extensión a línea media con bordes indefinidos (Figura 3C). En la reconstruc-

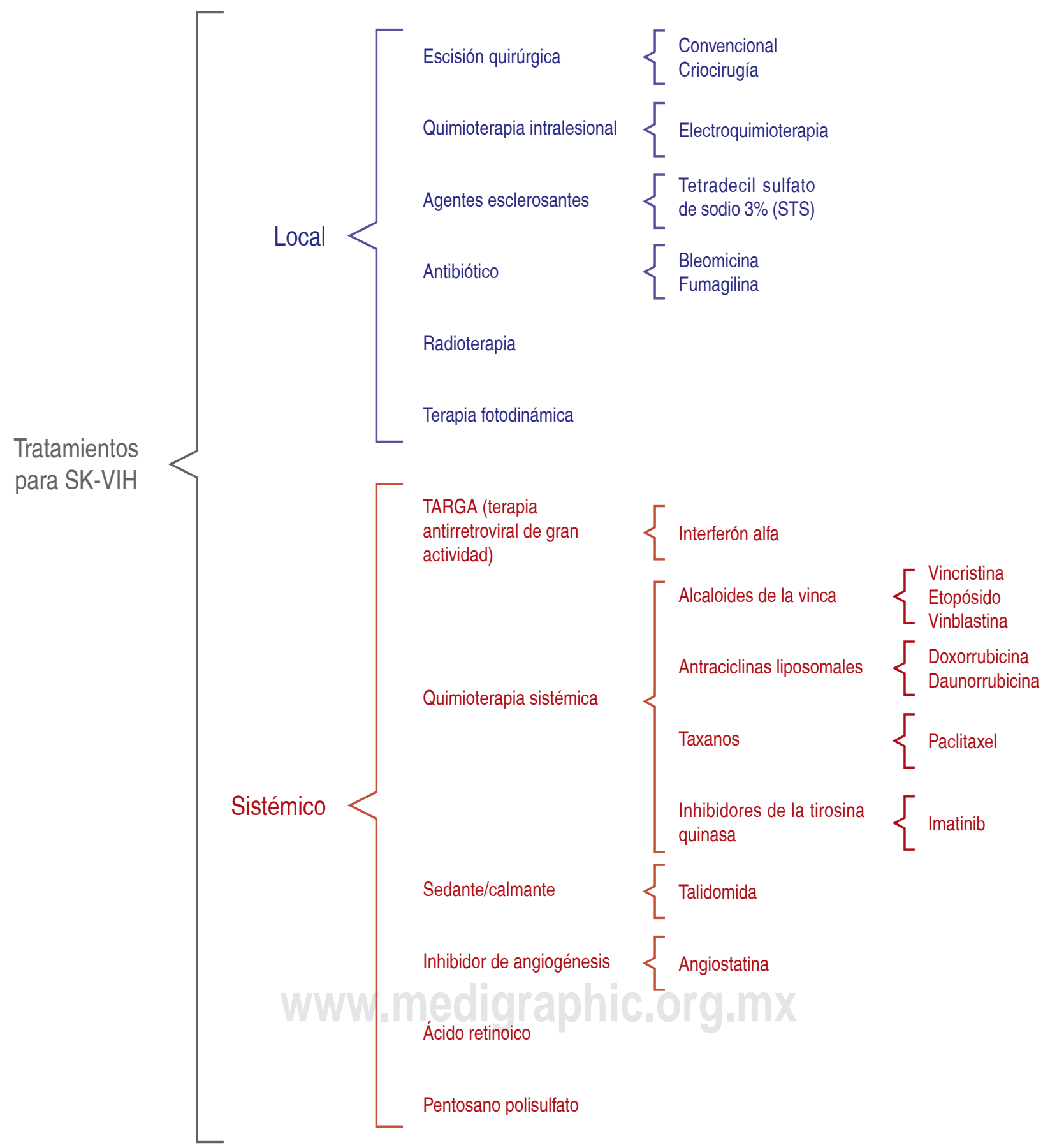

Figura 2: Tipos de tratamientos para el SK-VIH. Divididos en locales (cuadro azul) y sistémicos (cuadro rojo). ${ }^{35-45}$ 

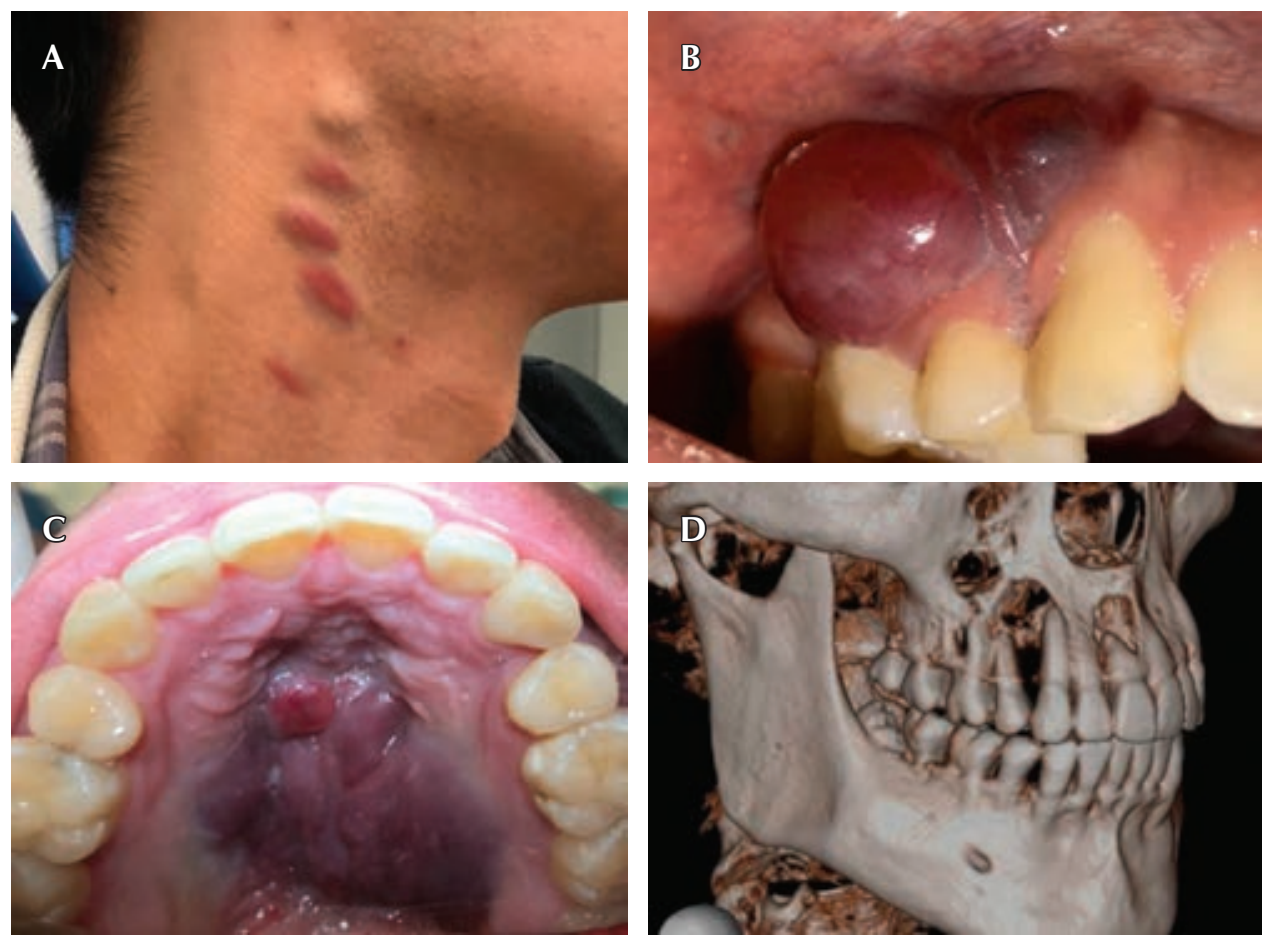

Figura 3:

A) Lesiones extraorales pápulovioláceas en cuello. B) Aumento de volumen vestibular. C) Lesión en paladar duro de bordes indefinidos con extensión a línea media. D) Reconstrucción 3D en la que se muestra la reabsorción ósea y el desplazo de las piezas adyacentes.

ción tridimensional con imagenología CBCT (Tomografía Computarizada de Haz Cónico, por sus siglas en inglés) se puede notar el desplazamiento de raíces dentales adyacentes y resorción de la cortical ósea vestibular en el área de la lesión (Figura 3D).

A partir de las manifestaciones clínicas, la examinación radiográfica y la historia clínica del paciente se establece el diagnóstico de SK. Se le informa al paciente que las manifestaciones presentes en boca están relacionadas con su enfermedad de base y no son de origen odontogénico. Por tanto, se contacta con su médico tratante para establecer un manejo multidisciplinario.

Debido al estado de supresión inmunológica del paciente y la probabilidad de infección de los tumores presentes en boca, se establecen medidas profilácticas para eliminar factores que comprometan su salud. Entre éstas están: realización de «profilaxis» dental cada seis meses para el control de índice del biofilm de placa bacteriana, descartar problemas cariogénicos y la extracción de terceros molares inferiores retenidos.

\section{DISCUSIÓN}

La infección por el VIH continúa siendo una de las causas principales de morbimortalidad a nivel mundial. ${ }^{46,47}$ En el caso de México, la Secretaría de Salud y el Centro
Nacional para la Prevención y el Control del VIH y SIDA (CENSIDA) reportaron 172,390 casos de VIH y SIDA vivos en 2019, con 7,668 nuevos casos diagnosticados en el mismo año. ${ }^{48}$

A pesar de esto, la terapia con fármacos antirretrovirales (TARGA) ha logrado disminuir la incidencia de pacientes con $\mathrm{VIH} / \mathrm{SIDA}$, así como las manifestaciones orales, por lo que patologías como el SK se ve con menor frecuencia. ${ }^{8,19}$ Lo anterior se debe también al incremento de la educación sexual, así como la concientización de los múltiples vectores de contagio del VIH.

Por esto, es de suma importancia la correcta anamnesis en la historia clínica, la inspección extra- e intraoral y el conocimiento de las manifestaciones clínicas de esta patología para el correcto diagnóstico y manejo odontológico.

Coogan y colaboradores mencionan que las manifestaciones orales son el indicador más temprano de la infección por el VIH y su progresión al SIDA. ${ }^{49}$ Ottria y sus colegas refieren que es la neoplasia más común asociada al síndrome. ${ }^{50}$

En la mayoría de la bibliografía consultada se concluyó que las zonas más comunes de aparición son en paladar duro, la encía insertada y el dorso de la lengua. ${ }^{11}$ El aspecto es de máculas eritematosas de color rojizo-violáceo poco circunscritas, ${ }^{13}$ variando en tamaño de 5 a $22 \mathrm{~mm} .{ }^{15}$ 
Como manifestaciones orales se presenta sangrado gingival, movilidad y pérdida dental, interferencia fonética, de la masticación y retención dentaria. ${ }^{18}$

En el caso presentado, el paciente fue sometido en dos ocasiones a tratamientos inadecuados por desconocimiento de las manifestaciones, lo que dio como resultado el avance y crecimiento de la patología, ya que no se identificó en una etapa más oportuna.

Asimismo, es indispensable considerar el tema de las barreras de bioseguridad por parte del odontólogo. De acuerdo con un estudio realizado en Nuevo León por el Tecnológico de Monterrey, se estudió la percepción y actitudes que toman los pacientes $\mathrm{VIH}$ positivos ante los servicios odontológicos. Los resultados refieren que 98\% de los pacientes no mencionan que son seropositivos previo a su atención dental. El 92\% consideró que estaban en su derecho de no mencionar su condición sistémica en la anamnesis. El 89\% cree que si lo mencionaban les negarían la atención dental. Y 79\% de la muestra cree que no pueden transmitir el $\mathrm{VIH}$ al odontólogo durante la atención dental. ${ }^{51}$

Es aquí donde se resalta la importancia del conocimiento de todas las características de la patología para tener un diagnóstico oportuno que vaya en pro de la salud del paciente y en caso de que éste no mencione o no esté consciente de su padecimiento, el odontólogo y el personal clínico odontológico esté protegido al momento de realizar la atención dental evitando un contagio accidental.

Para esto es necesario conocer las normativas correspondientes a su país que hablen del control de infecciones y bioseguridad. En el caso de México, la Norma Oficial Mexicana NOM-013-SSA2-2006 menciona lo pertinente a este rubro. En ésta se indica el utilizar las barreras de bioseguridad como el uniforme clínico (gorro, lentes o máscara, guantes de látex, cubrebocas y bata desechable), protectores de la unidad dental, manejo correcto de los punzocortantes y protocolos de esterilización cuando el instrumental se utiliza en pacientes $\mathrm{VIH}$ positivos $\left(180^{\circ} / 1 \mathrm{~h}\right.$ con calor seco, $121^{\circ} /$ 20 min o $56^{\circ} / 30$ min con vapor). ${ }^{52}$

\section{CONCLUSIÓN}

En la actualidad, se ha reportado una reducción en la incidencia del sarcoma de Kaposi asociado al VIH/SIDA (SK-VIH/SIDA) desde mediados de 1990 y se considera ahora una neoplasia oral poco frecuente. ${ }^{17}$ Gracias a la aparición de los TARGA, se ha visto una disminución de $79 \%$ de los casos de SK en un periodo inferior a dos décadas e incluso la han llamado «una neoplasia olvidada». ${ }^{19}$ Por lo tanto, todos los odontólogos deben tener los conocimientos necesarios para identificar y diagnosticar esta neoplasia con el objetivo de brindar un diagnóstico temprano y tratamiento oportuno.

El SK-VIH/SIDA presenta gran variedad de formas clínicas, desde lesiones maculopapulares hasta tumoraciones multifocales. Debido a la diversidad morfológica de la neoplasia es común confundirla con gran cantidad de patologías orales como gingivitis, periodontitis, abscesos, infecciones odontogénicas u otros tipos de tumores.

Aunado a esto, se presenta la situación que muchos pacientes desconocen o no mencionan su condición seropositiva. Por ello, es indispensable conocer las indicaciones de las normativas de control de infección y bioseguridad, así como las guías de atención estomatológica en pacientes con $\mathrm{VIH}$.

Al mantener las barreras de bioseguridad y conocer las características de la patología podremos dar un adecuado manejo odontológico al SK-VIH/SIDA.

\section{BIBLIOGRAFÍA}

1. Feller L, Wood NH, Lemmer J. HIV-associated Kaposi sarcoma: pathogenic mechanisms. Oral Surg Oral Med Oral Pathol Oral Radiol Endod. 2007; 104 (4): 521-529.

2. Whitby D, Howard MR, Tenant-Flowers M, Brink NS, Copas A, Boshoff $\mathrm{C}$ et al. Detection of Kaposi sarcoma associated herpesvirus in peripheral blood of HIV-infected individuals and progression to Kaposi's sarcoma. Lancet. 1995; 346 (8978): 799-802.

3. US Department of Health and Human Services. AIDS info Glossary of HIV/AIDS-Related Terms. 2008. Disponible en: https://aidsinfo. nih.gov/contentfiles/glossaryhivrelatedterms_english.pdf

4. Antmam K, Chang Y. Kaposi's sarcoma. N Engl J Med. 2000; 342 (14): 1027-1038.

5. Cancela RB, Álvarez-Gallegos V. Sarcoma de Kaposi clásico. Rev Cent Dermatol Pascua. 2000; 9 (1): 6-10.

6. Enríquez AR. Sarcoma de Kaposi?: revisión de la literatura e ilustración de un caso. Acta Médica Grup Ángeles. 2013; 11 (1): 23-31.

7. Najafi F, Kafaie P, Neamatzadeh H. Treatment of an early Kaposi 's sarcoma case post kidney transplantation by sirolimus?: a case report. Acta Med Iran. 2016; 55 (2): 139-141.

8. Lima CT, Araújo PSR, Teixeira HM, Santos JBD, Silveira VMD. Clinical and laboratory characteristics, staging, and outcomes of individuals with AIDS-associated Kaposi's sarcoma at an university hospital. An Bras Dermatol. 2017; 92 (2): 172-176.

9. Ficarra G, Berson AM, Silverman S. Kaposi's sarcoma of the oral cavity?: a study of 134 patients with a review of the pathogenesis, epidemiology, clinical aspects, and treatment. Oral Surg. 1988; 66 (5): 543-550.

10. Epstein JB, Cabay RJ, Glick M. Oral malignancies in HIV disease: changes in disease presentation, increasing understanding of molecular pathogenesis, and current management. Oral Surg Oral Med Oral Pathol Oral Radiol Endod. 2005; 100 (5): 571-578. 
11. Epstein JB. Management of oral Kaposi's sarcoma and a proposal for clinical staging. Oral Dis. 1997; 3 (1): 124-128.

12. Fatahzadeh M, Schwartz RA, Edin F. Oral Kaposi's sarcoma?: a review and update. Int J Dermatol. 2013; 52 (1): 666-672.

13. Petit JC, Ripamonti U, Hillef J. Progressive changes of Kaposi's sarcoma of the gingiva and palate. J Periodontol. 1986; 57 (3): 159-163.

14. Chaurasia A. CD4+ counts and oral lesions in HIV infected/AIDS patients- an Indian perspective. Juniper Online J Case Stud. 2017; 1 (5): 4-7.

15. Agaimy A, Mueller SK, Harrer T, Bauer S, Thompson LDR. Head and neck Kaposi sarcoma?: clinicopathological analysis of 11 cases. Head Neck Pathol [Internet]. 2018; 12 (4): 511-516. Available from: http://dx.doi.org/10.1007/s12105-018-0902-x

16. Lausten LL, Ferguson BL, Barker BF, Cobb CM. Case report oral Kaposi sarcoma associated with severe alveolar bone loss?: case report and review of the literature case report. J Periodontol. 2003; 74 (11): 1668-1675.

17. Kalpidis CDR, Lysitsa SN, Lombardi T, Kolokotronis AE, Antoniades DZ, Samson J. Gingival involvement in a case series of patients with acquired immunodeficiency syndrome-related kaposi sarcoma. J Periodontol. 2006; 77 (3): 523-533.

18. Reichart PA. Oral manifestations in HIV infection: Fungal and bacterial infections, Kaposi's sarcoma. Med Microbiol Immunol. 2003; 192 (3): 165-169.

19. Maya A, Segovia S, Madrigal S, Abrego I, Salas-Alanis JC. Sarcoma de Kaposi en región oral y maxilofacial, una neoplasia olvidada. Rev Esp Cir Oral y Maxilofac. 2018; 40 (1): 22-26.

20. Kedes DH, Ganem D. Sensitivity of Kaposi's sarcoma-associated herpesvirus replication to antiviral drugs. Phys Today. 1971; 24 (5): 2082-2086.

21. Casper C, Krantz EM, Corey L, Kuntz R, Wang J, Selke S et al. Valganciclovir for suppression of human herpesvirus 8 replication: a randomized, double-blind, placebo-controlled, crossover trial. J Infect Dis. 2009; 198 (1): 23-30.

22. Riva G, Barozzi P, Torelli G, Luppi M. Immunological and inflammatory features of Kaposi's sarcoma and other Kaposi's sarcoma-associated herpesvirus/human herpesvirus 8-associated neoplasias. AIDS Rev. 2010; 12 (1): 40-51.

23. Schulz TF. The pleiotropic effects of Kaposi's sarcoma herpesvirus. J Pathol. 2006; 208 (1): 187-198.

24. Ariza-heredia EJ, Razonable RR. Human herpes virus 8 in solid organ transplantation. Transplantation. 2011; 92 (8): 837-844.

25. Heyrman B, Becker A De, Schots R. A case report of immunosuppression? related Kaposi's sarcoma after autologous stem cell transplantation. BMC Res Notes. 2016; 9 (188): 1-5.

26. Instituto Nacional del Cáncer. Targa. Diccionario de Cáncer. 2020. p. 1. Disponible en: https://www.cancer.gov/espanol/publicaciones/ diccionario/def/targa

27. By Jmarchn, HIV-drug-classes-es [Figura 2] (https://commons. wikimedia.org/wiki/File:HIV-drug-classes-es.svg), https:// creativecommons.org/licenses/by-sa/3.0/legalcode

28. Pantanowitz L, Khammissa RA, Lemmer J, Feller L. Oral HIVassociated Kaposi sarcoma. J oral Pathol Med. 2013; 42: 201277.

29. Feller L, Lemmer J. Oral Kaposi sarcoma. In: Pantanowitz L, Stebbing J, Dezube BJ, eds. Kaposi sarcoma: a model of oncogenesis. Kerala, India: Research Signpost; 2010. pp. 295-303.

30. Reyes-Corcho A, Bouza-Jiménez Y. Síndrome de reconstitución inmunológica asociado al virus de la inmunodeficiencia humana y sida. Estado del arte. Enferm Infecc Microbiol Clin. 2010; 28 (2): 110-121.
31. Shelburne SA, Montes M, Hamill RJ. Immune reconstitution inflammatory syndrome?: more answers, more questions. J Antimicrob Chemother. 2018; 57 (1): 167-170.

32. Stone SF, Price P, French MA. Immune restoration disease: a consequence of dysregulated immune responses after HAART. Curr HIV Res. 2004; 2 (3): 235-242.

33. Manandhar R, Shrestha N, Butlin CR, Mycobacterial PWR, Leprosy A. High levels of inflammatory cytokines are associated with poor clinical response to steroid treatment and recurrent episodes of type 1 reactions in leprosy. Clin Exp Immunol. 2002; 128: 333-338.

34. Hirsch HH, Kaufmann G, Sendi P, Battegay M. Immune reconstitution in HIV-infected patients. Clin Infect Dis. 2004; 38 (8): 1159-1166.

35. Montoya E, Harold J, Leguízamo E, Nayive D. Manifestaciones orales de la infección por VIH/sida en niños y adolescentes?: aspectos clínicos, epidemiología y pautas de tratamiento. Univ Odontológica. 2011; 30 (64): 37-50.

36. Schöfer H. Kaposi-sarkom-Update 1997. In: Garbe C, Rassner G (eds.). Dermatologie. Heidelberg: Springer, Berlin; 1998.

37. Shirlaw PJ, Chikte U, Macphail L, Croser D, Reichart P. Oral and dental care and treatment protocols for the management of HIVinfected patients. Oral Dis. 2002; 8 (2): 136-143.

38. Tappero JW, Conant MA, Wolfe SF, Berger TG. Continuing medical education Kaposi's sarcoma. J Am Acad Dermatol [Internet]. 1993; 28 (3): 371-395. Available from: http://dx.doi.org/10.1016/01909622(93)70057-Z

39. Ramírez-Amador V, Esquivel-Pedraza L, Lozada-Nur F, De la RosaGarcía E, Volkow-Fernández P, Súchil-Bernal L et al. Intralesional vinblastine vs. $3 \%$ sodium tetradecyl sulfate for the treatment of oral Kaposi's sarcoma. A double blind, randomized clinical trial. Oral Oncol. 2002; 38 (5): 460-467.

40. Merino JE, Janina R, Valdez L. Escleroterapia. Rev Cent Dermatol Pascua. 2008; 17 (1): 11-18.

41. Scully C, Langdon J, Evans J. Marathon of eponyms?: 11 Kaposi sarcoma. Oral Dis. 2010; 16 (1): 402-403.

42. Pinheiro S, Teno T, Maria C, Ribeiro B, Lea JC, Pomarico I et al. Oral manifestations in human immunodeficiency virus infected children in highly active antiretroviral therapy era. Oral Pathol Med. 2009; 38 (1): 613-622.

43. Reichart PA. Oral pathology of acquired immunodeficiency syndrome and oro-facial Kaposi's sarcoma. In: Seifert G (eds.). Oral pathology. Current topics in pathology. Vol 90. Heidelberg: Springer, Berlin; 1996.

44. Yarchoan R. Therapy for Kaposi's sarcoma: recent advances and experimental approaches. J Acquir Immune Defic Syndr. 1999; 21 Suppl 1: S66-S73.

45. Warnock DW. Amphotericin B: an introduction. J Antimicrob Chemother. 1991; 28 Suppl B: 27-38.

46. Girum T, Wasie A, Worku A. Trend of HIV/AIDS for the last 26 years and predicting achievement of the 90-90-90 HIV prevention targets by 2020 in ethiopia: a time series analysis. BMC Infect Dis. 2018; 18 (1): 1-10.

47. Ortblad KF, Lozano R, Murray CJL. The burden of HIV: Insights from the global burden of disease study 2010. AIDS. 2013; 27 (13): 2003-2017.

48. Centro Nacional para la Prevención y el Control del VIH y el sida. Vigilancia epidemiológica de casos de VIH/SIDA en México. Registro Nacional de Casos de sida. Actualización al 2019.

49. Coogan MM, Greenspan J, Challacombe SJ. Oral lesions in infection with human immunodeficiency virus. Bull World Health Organ. 2005; 83 (9): 700-706. 
50. Ottria L, Lauritano D, Oberti L, Candotto V, Cura F, Tagliabue A et al. Prevalence of HIV-related oral manifestations and their association with HAART and CD4 + T cell count: a review. J Biol Regul Homeost Agents. 2018; 32 (2 Suppl. 1): 51-59.

51. Elizondo JE, Treviño AC, Violant D. Dentistry and HIV/AIDS related stigma. Rev Saude Publica. 2015; 49. pii: S003489102015000100263.

52. Garza AMG. Control de infecciones y bioseguridad en odontología. (2a ed. Ciudad de México: El Manual Moderno; 2016.

\section{Correspondencia:}

\section{Dr. Gustavo Pedro Díaz Colmenares}

Centro Académico de Atención Odontológica (CAAD).

Av. Morones Prieto Núm. 3000 Pte.,

Col. Los Doctores, 64060,

Monterrey, NL, México.

Tel: 8124680002

E-mail: gus.d96@gmail.com 